\title{
Encapsulating of single quantum dots into polymer particles
}

\author{
Yan Gao • Sabrina Reischmann • Johannes Huber • \\ Tobias Hanke • Rudolf Bratschitsch • \\ Alfred Leitenstorfer $\cdot$ Stefan Mecking
}

\begin{abstract}
Single semiconductor quantum dots were embedded into polymer particles with diameters below $0.1 \mu \mathrm{m}$ by an emulsion polymerization procedure or via a secondary dispersion approach. The photoluminescence properties of the nanocrystals are retained upon encapsulation, as demonstrated by fluorescence confocal microscopy.
\end{abstract}

Keywords Quantum dots · Emulsion polymerization . Hybrid particles · Polymerization · Fluorescence

\begin{tabular}{ll}
\multicolumn{2}{l}{ Abbreviations } \\
QDs & Quantum dots \\
SDS & Sodium dodecyl sulfate \\
MMA & methyl methacrylate \\
AIBN & 2,2-azobisisobutyronitrile \\
DLS & Dynamic light scattering \\
AFM & atomic force microscopy
\end{tabular}

\section{Introduction}

Hybrid organic-inorganic particles are of widespread interest [1]. The incorporation of inorganic nanoparticles in larger polymer micro- or nanoparticles will provide them with the colloidal properties of the latter, e.g., dispersability

Y. Gao $\cdot$ S. Reischmann $\cdot$ J. Huber $\cdot$ S. Mecking $(\bowtie)$

Chemical Materials Science, Department of Chemistry,

University of Konstanz,

Constance, Germany

e-mail: stefan.mecking@uni-konstanz.de

T. Hanke $\cdot$ R. Bratschitsch $\cdot$ A. Leitenstorfer

Modern Optics and Quantum Electronics, Department of Physics,

University of Konstanz,

Constance, Germany in a given medium and adsorption behavior towards a surface. The inorganic nanoparticles can be protected from their environment and from damage incurred upon mechanical manipulation. Manipulation may also be facilitated by the "size enlargement" occurring by the incorporation in the larger polymer particles.

Among various methods for the preparation of nanocomposite particles, heterophase polymerization has proven particularly versatile [2-6]. In order to provide compatibility with an organic phase (e.g., solvent, monomer, or polymer), inorganic nanoparticles with a bound or physisorbed organic shell are employed. Due to a limited miscibility of polymers with this organic shell, aggregation of the inorganic nanoparticles frequently occurs upon polymer formation $[6,7]$. The preparation of composite particles with nonaggregated inorganic nanoparticles is challenging.

Inorganic semiconductor quantum dots (QDs) possess unique optical properties, namely size-dependent tunable emission, photostability, broad absorption spectra, and narrow emission spectra [8-10]. In order to enhance the fluorescence efficiency of QDs, particularly of CdSe nanocrystals, much effort has been devoted to protect their surface by a thin shell of an inorganic material with a wider band gap such as ZnS [11, 12]. The QDs remain sensitive, however, towards their environment and to mechanical damage upon manipulation [13-15]. The incorporation of QDs into inorganic or polymer microspheres can enhance their photostability and biocompatibility [16-19]. Welldefined monodisperse core-shell polymer particles, which form colloidal crystals, with core-embedded QDs have been prepared by a miniemulsion procedure [6].

For efficient single photon sources, single QDs need to be incorporated in tailored dielectric [20] and metallic [21] environments. Studies of the control of the interaction between the light field and quantum dots by metallic 
Fig. 1 TEM (a) and AFM (b) image of particles prepared by secondary dispersion (entry 1-2)
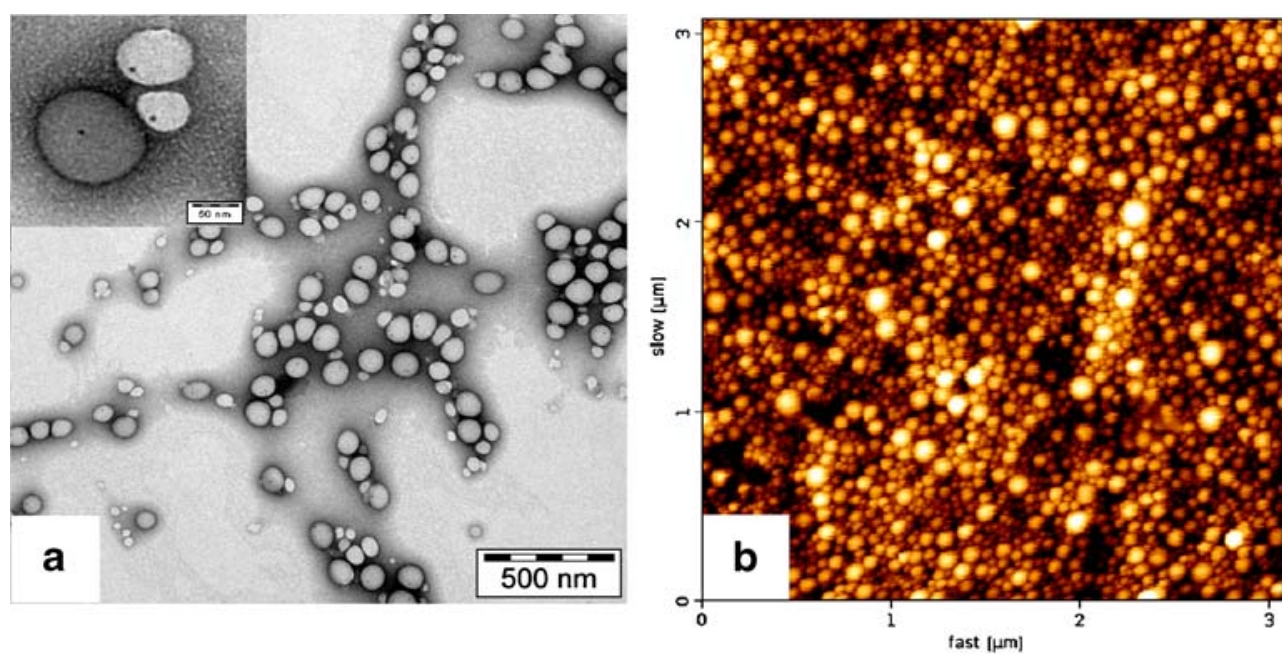

antenna structures require placement of a single QD in the much larger antenna structure. An approach to facilitate this placement is size enlargement by incorporation in a polymer particle. Note that in these studies, single positions in the antenna structure are addressed individually, and the substrate can be scanned for suitable arrangements, such that the latter need not be perfect for the entire sample. However, the quantum dots must be intact and present largely as individual, nonaggregated entities. Our studies of the embedding of QDs in polymer particles, therefore, focused on the preferential incorporation of single nonaggregated QDs. Emulsion polymerization of methyl methacrylate (MMA) was employed. As an alternative, a secondary $[18,22]$ dispersion approach is also reported.

\section{Experimental}

Materials Sodium dodecyl sulfate (SDS), MMA, and 2,2azobisisobutyronitrile (AIBN) were purchased from Fluka. MMA was distilled under reduced pressure to remove inhibitor and stored under argon at $4{ }^{\circ} \mathrm{C}$ prior to use. Deionized water was distilled under nitrogen before use.
Poly(butyl methacrylate-co-methyl methacrylate) with $\mathrm{M}_{\mathrm{w}} 10^{5} \mathrm{~g} \mathrm{~mol}^{-1}$ and $\mathrm{T}_{\mathrm{g}} 64^{\circ} \mathrm{C}$ was purchased from Aldrich. CdSe $(\lambda=610 \mathrm{~nm})$ and $\mathrm{CdSe} / \mathrm{ZnS}(\lambda=640 \mathrm{~nm})$ QDs, which are stabilized with octylamine and dispersed in toluene, were acquired from the nn-labs company and used as received. According to the supplier, the mass concentration for CdSe is $5 \mathrm{mg} \mathrm{mL}^{-1}$, corresponding to a molar concentration of nanocrystals of $3.0 \times 10^{-5} \mathrm{~mol} \mathrm{~L}^{-1}$ (molar concentrations determined applying Beer's law and experimentally determined extinction coefficients). CdSe/ZnS solutions with two different concentrations were employed, 2 and $5 \mathrm{mg} \mathrm{mL}^{-1}$, respectively, corresponding to molar concentrations of $1.0 \times 10^{-5}$ and $2.65 \times 10^{-5} \mathrm{~mol} \mathrm{~L}^{-1}$. Calculated number densities of the three samples are $1.8 \times$ $10^{19}, 6 \times 10^{18}$, and $1.6 \times 10^{19} \mathrm{~L}^{-1}$, respectively.

Encapsulation of quantum dots in P(BMA-co-MMA) by secondary dispersion Two milliliters of toluene solution containing a variable amount of QDs ranging from 0.4 to $1.6 \mathrm{mg}$ were mixed with $0.4 \mathrm{~g}$ of polymer. The solution was mixed thoroughly by shaking overnight. A solution of $10 \mathrm{mg}$ SDS in $20 \mathrm{~mL}$ of water was added. The mixture was stirred for 5 min under an argon atmosphere. Then, it was

Table 1 Results of secondary dispersion

\begin{tabular}{llcc}
\hline Entry & $\begin{array}{l}\text { Volume QD } \\
\text { solution employed }\end{array}$ & Particle size ${ }^{\mathrm{b}}$ (PDI) & $\begin{array}{l}\text { Portion of QD-containing } \\
\text { polymer particles }\end{array}$ \\
\hline $1-1$ & $0.2 \mathrm{~mL}$ & $111 \mathrm{~nm}(0.08)$ & $18 \%$ \\
$1-2$ & $0.4 \mathrm{~mL}$ & $107 \mathrm{~nm}(0.08)$ & $24 \%$ \\
$1-3$ & $0.8 \mathrm{~mL}$ & $108 \mathrm{~nm}(0.09)$ & aggregates \\
\hline
\end{tabular}

$0.4 \mathrm{~g}$ PBMA-co-PMMA, $20 \mathrm{~mL}$ of water, $10 \mathrm{mg}$ of SDS, total volume of toluene: $2 \mathrm{~mL}$.

a Toluene solution of $\mathrm{CdSe} / \mathrm{ZnS}$ with a concentration of $2 \mathrm{mg} \mathrm{mL}^{-1}\left(1.1 \times 10^{-5} \mathrm{~mol} \mathrm{~L}^{-1} ; 6.4 \times 10^{18}\right.$ particles $\left.\mathrm{L}^{-1}\right)$.

${ }^{\mathrm{b}}$ Volume average particle size and size distribution determined by DLS. 


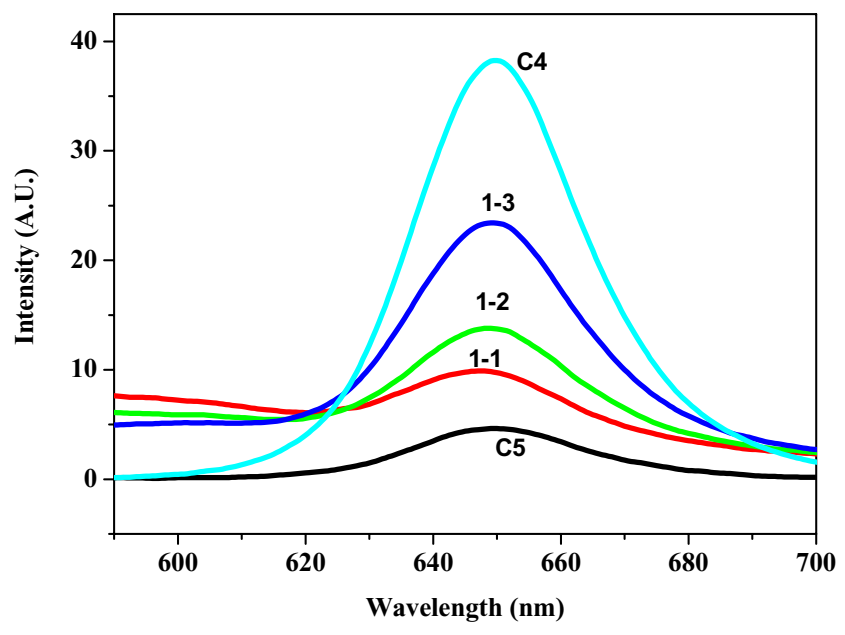

Fig. 2 Fluorescence spectra of polymer-encapsulated CdSe/ZnS from secondary dispersion (entries $1-1$ to $1-3$ ). Toluene solution of $\mathrm{CdSe} /$ $\mathrm{ZnS}$ for comparison (particle number density $3.8 \times 10^{15} \mathrm{~L}^{-1}(C 5)$ and $1.5 \times 10^{16} \mathrm{~L}^{-1}(C 4)$

ultrasonicated for 10 min (Bandelin HD2200 with KE76 tip, operated at $120 \mathrm{~W})$. The solvent was removed by stirring overnight and then under reduced pressure (20 mbar) for $5 \mathrm{~h}$, during which additional $2 \mathrm{~mL}$ of water were injected.

Encapsulation of quantum dots in PMMA particles by polymerization in emulsion A typical procedure was as follows: under an argon atmosphere, $10 \mathrm{mg}$ of SDS were dissolved in $80 \mathrm{~mL}$ of degassed water, followed by addition of $1 \mathrm{~mL}$ of MMA; $10 \mathrm{mg}$ of AIBN were dissolved in a variable volume of toluene solution of QDs ranging from 0.25 to $1 \mathrm{~mL}$ (corresponding to 0.5 to $2 \mathrm{mg}$ of QDs; also cf. Table 2). The two solutions were mixed and stirred for $10 \mathrm{~min}$. The biphasic mixture was ultrasonicated under an argon atmosphere for 5 min (Bandelin HD2200 with KE76 tip, operated at $120 \mathrm{~W}$ ). After transferring the resulting miniemulsion to a $250 \mathrm{~mL}$ four-necked glass reactor equipped with a mechanical stirrer and a condenser, the polymerization was continued for $6 \mathrm{~h}$ at $80^{\circ} \mathrm{C}$. The resulting dispersion was stirred in an open vessel overnight to largely remove the toluene.

Characterization Dynamic light scattering (DLS) was performed on a Malvern Nano-ZS ZEN 3600 particle sizer (173 ${ }^{\circ}$ back scattering). The autocorrelation function was analyzed using the Malvern dispersion technology software 5.00 algorithm to obtain volume weighted particle size distributions. Atomic force microscopy (AFM) was performed on a JPK NanoWizard instrument in intermittent contact mode. Electron microscopy was performed on a Zeiss Libra 120 transmission electron microscope (TEM); $15 \mu \mathrm{L}$ of the diluted dispersion with a concentration of ca. 0.02 wt.\% were applied to a carbon-coated copper grid and left to dry overnight. Fluorescence spectra of the solution were obtained with a Perkin Elmer Luminescence Spectrometer LS50B with $390 \mathrm{~nm}$ excitation. The microphotoluminescence investigation was carried out in reflection geometry using a Nd:YAG laser at a wavelength of $532 \mathrm{~nm}$. It was focused with a microscope objective (Mitutoyo M Plan APO 100, NA 0.7) onto the sample, resulting in a spatial resolution of $0.8 \mu \mathrm{m}$.

\section{Results and discussion}

\section{Secondary dispersions}

The preparation of polymer particles with encapsulation of the inorganic nanoparticles was studied employing a premade polymer. In order to reduce the viscosity of the polymer to enable its dissipation into small particles, and also to enable mixing of the polymer and the inorganic nanoparticles, both were dissolved in toluene as a common solvent. Dispersion of this solution in an aqueous surfactant solution by shear generated by ultrasonication, followed by solvent removal afforded poly(butyl methacrylate-co-methyl methacrylate) particles with encapsulated $\mathrm{CdSe} / \mathrm{ZnS}$ quantum dots (Fig. 1 and Table 1). Average polymer particle sizes
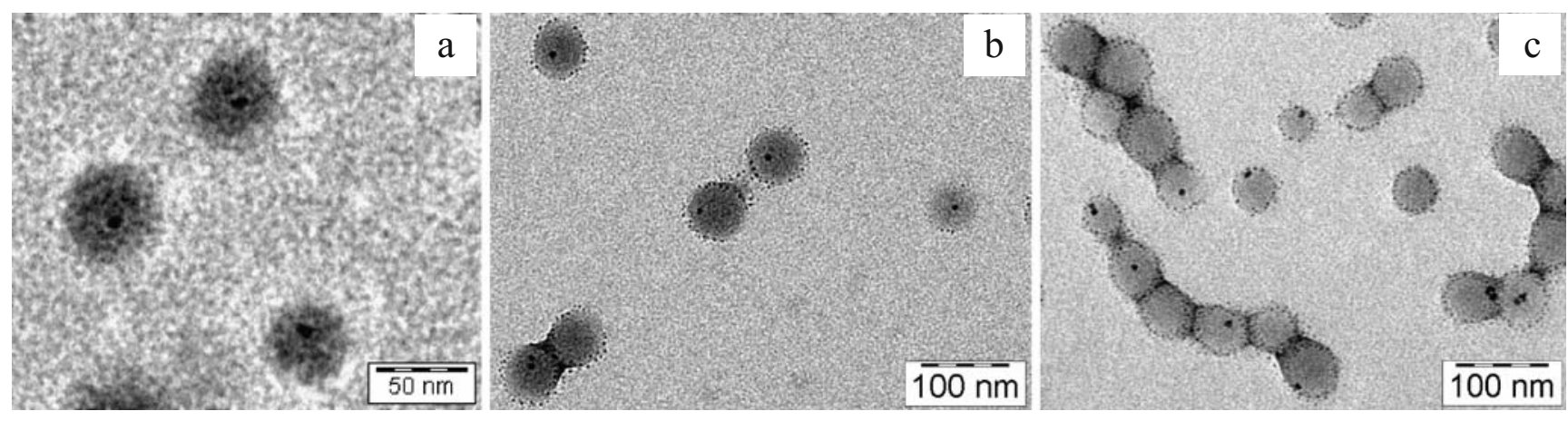

Fig. 3 TEM images of PMMA-encapsulated quantum dots prepared by polymerization in emulsion. (a): entry 2-2, (b): entry 2-5, (c) entry 2-7 
Table 2 Results of polymerization in emulsion

\begin{tabular}{|c|c|c|c|c|c|c|c|}
\hline Entry & QD type & $\begin{array}{l}\text { Volume QD } \\
\text { solution employed }^{\text {a) }}\end{array}$ & $\begin{array}{l}\text { Amount } \\
\text { of SDS }\end{array}$ & $\begin{array}{l}\text { Particle } \\
\text { size }^{\mathrm{b}} \text { (PDI) }\end{array}$ & $\begin{array}{l}\text { QD particle } \\
\text { number density }^{\mathrm{c}}\end{array}$ & $\begin{array}{l}\text { Polymer particle } \\
\text { number density }\end{array}$ & $\begin{array}{l}\text { Portion of QD-containing } \\
\text { polymer particles }\end{array}$ \\
\hline $2-1$ & & - & $10 \mathrm{mg}$ & $66(0.03)$ & - & $7 \times 10^{16}$ & - \\
\hline $2-2$ & $\mathrm{CdSe}$ & $0.2 \mathrm{~mL}$ & $10 \mathrm{mg}$ & $62(0.03)$ & $5 \times 10^{16}$ & $9 \times 10^{16}$ & $54 \%$ \\
\hline $2-3$ & $\mathrm{CdSe}$ & $0.5 \mathrm{~mL}$ & $10 \mathrm{mg}$ & $47(0.04)$ & $1 \times 10^{17}$ & $2 \times 10^{17}$ & n.d. \\
\hline $2-4$ & $\mathrm{CdSe} / \mathrm{ZnS}$ & $0.25 \mathrm{~mL}$ & $3 \mathrm{mg}$ & $75(0.04)$ & $2 \times 10^{16}$ & $6 \times 10^{16}$ & $33 \%$ \\
\hline $2-5$ & $\mathrm{CdSe} / \mathrm{ZnS}$ & $0.5 \mathrm{~mL}$ & $6 \mathrm{mg}$ & $68(0.03)$ & $4 \times 10^{16}$ & $7 \times 10^{16}$ & $48 \%$ \\
\hline $2-6$ & $\mathrm{CdSe} / \mathrm{ZnS}$ & $1.0 \mathrm{~mL}$ & $10 \mathrm{mg}$ & $41(0.12)$ & $8 \times 10^{16}$ & $3 \times 10^{17}$ & aggregates \\
\hline $2-7$ & $\mathrm{CdSe} / \mathrm{ZnS}$ & $0.4 \mathrm{~mL}$ & $10 \mathrm{mg}$ & $66(0.04)$ & $8 \times 10^{16}$ & $7 \times 10^{16}$ & $40 \%$ \\
\hline
\end{tabular}

One milliliter of MMA, $80 \mathrm{~mL}$ of water, $10 \mathrm{mg}$ of AIBN (entry 2-4: $0.5 \mathrm{~mL}$ of MMA, $5 \mathrm{mg}$ of AIBN).

${ }^{a}$ toluene solution of CdSe with a concentration of $5 \mathrm{mg} \mathrm{mL}^{-1}\left(3 \times 10^{-5} \mathrm{~mol} \mathrm{~L}^{-1} ; 1.8 \times 10^{19}\right.$ particles $\left.\mathrm{L}^{-1}\right)$ and of CdSe/ZnS with a concentration of $2 \mathrm{mg} \mathrm{mL}^{-1}\left(1.06 \times 10^{-5} \mathrm{~mol} \mathrm{~L}^{-1} ; 6.4 \times 10^{18}\right.$ particles $\left.\mathrm{L}^{-1}\right)$, respectively (entry $2-7$ : CdSe/ZnS $5 \mathrm{mg} \mathrm{mL}^{-1}\left(3 \times 10^{-5} \mathrm{~mol} \mathrm{~L}^{-1} ; 1.8 \times 10^{19}\right.$ particles $\left.\mathrm{L}^{-1}\right)$ ).

${ }^{\mathrm{b}}$ Volume average particle size and size distribution determined by DLS.

${ }^{\mathrm{c}}$ Particle number density in the polymerization reaction mixture.

${ }^{\mathrm{d}}$ From particle size determined by DLS and polymer mass.

${ }^{\mathrm{e}}$ From TEM

of 70 to $80 \mathrm{~nm}$ (number weighted) were determined by TEM for entries $1-1$ and $1-2$. Note that the polymer particles are not exactly spherical, which may be related to the solvent removal step.

TEM (Fig. 1) reveals that the inorganic quantum dots are well dispersed in the polymer particles. The number of polymer particles containing $n=0,1,2$, or more quantum dots, as observed by TEM, agrees reasonably with an entirely statistical distribution ${ }^{1}$ within experimental error (entry 1-1: $n=1: 14 \%, n=2: 3 \%, n \geq 3:<1 \%$. Entry 1-2: $n=$ 1: $22 \%, n=2: 2 \%, n \geq 3:<1 \%$.). At a higher concentration of quantum dots (entry 1-3) aggregation occurred to a significant extent. A comparison of the particle number density of polymer particles (from DLS and the polymer mass content) and their aforementioned quantum dot content (from TEM) with the amount of quantum dots added in the preparation of the particles indicates that a large portion of quantum dots is not encapsulated in the polymer particles in the dispersion procedure but is lost likely due to agglomeration on the vessel surfaces.

The polymer-encapsulated $\mathrm{CdSe} / \mathrm{ZnS}$ particles retain their photoluminescence bands (Fig. 2). By comparison to toluene solutions of the same quantum dot particle number density as employed in the polymer particle preparation (referring to the volume of the entire reaction mixture), intensities are an order of magnitude lower. This can be accounted for largely by the aforementioned loss of quantum dots during polymer particle preparation.

\footnotetext{
${ }^{1}$ For a random placement of $\mathrm{x}$ balls into $\mathrm{N}$ baskets, with the probability of a given event being independent of previous events, the probability of a given basket containing $\mathrm{n}$ particles is $\mathrm{P}(n)=(1-$ $\left.\mathrm{N}^{-1}\right)^{\mathrm{x}-n}\left(\mathrm{~N}^{-1}\right)^{n} \mathrm{x} !((\mathrm{x}-n) !)^{-1}(n !)^{-1}$.
}

Dispersions from polymerization

The preparation of dispersions by polymerization in emulsion was studied. In order to introduce the hydrophobic quantum dots, a toluene solution of the latter was miniemulsified in an aqueous surfactant solution by ultrasonication. Under the polymerization conditions employed, the solubility of MMA monomer in the aqueous phase exceeds the concentration of the MMA present [23]. In the presence of the quantum dot-containing toluene droplets, the MMA will be partioned between the aqueous and the organic phase. Note that the solubility of toluene in water approaches, but does not quite exceed, the amount of

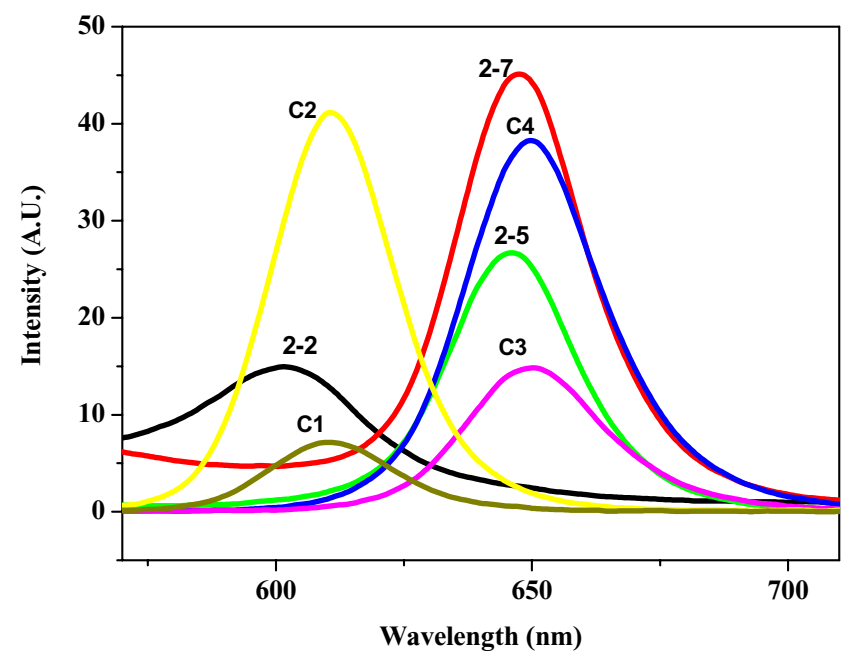

Fig. 4 Fluorescence spectra of polymer-encapsulated CdSe and CdSe/ $\mathrm{ZnS}$ from polymerization in emulsion (entry 2-2, Entry 2-5, and Entry 2-7). Toluene solution of CdSe (particle number density $2.2 \times$ $10^{16} \mathrm{~L}^{-1}(C l)$ and $\left.4.4 \times 10^{16} \mathrm{~L}^{-1}(C 2)\right)$ and $\mathrm{CdSe} / \mathrm{ZnS}$ (particle number density $0.75 \times 10^{16} \mathrm{~L}^{-1}(C 3)$ and $\left.1.5 \times 10^{16} \mathrm{~L}^{-1}(C 4)\right)$ for comparison 
Fig. 5 (a) Fluorescence confocal microscopy image of polymer particle-

encapsulated CdSe/ZnS QDs (from entry 1-1) on substrate. (b) Fluorescence spectrum of single polymer-encapsulated QD, (c) Spectrally resolved photoluminescence time trajectory of single polymerencapsulated QD. (d) Spectrally integrated photoluminescence time trajectory for the same particle. All measurements at room temperature
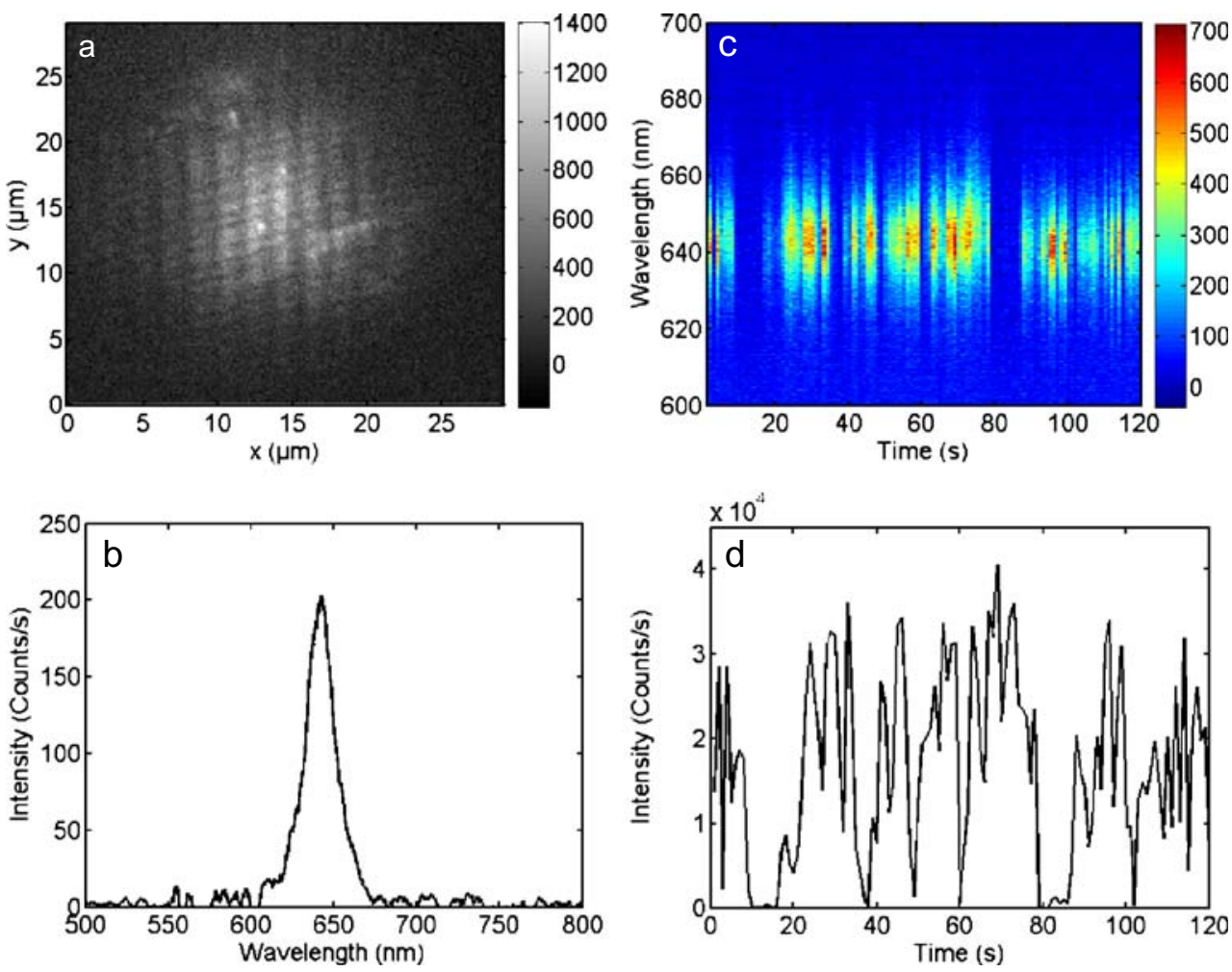

toluene present in some experiments (entry 2-4) [24]. In any case, the presence of the hydrophobic quantum dots should result in the existence of a part of the toluene as droplets.

The lipophilic initiator AIBN was employed, such that polymerization will occur in the droplets. The inorganic particles are well dispersed in the polymer particles obtained (Fig. 3 and Table 2. The origin of the numerous small dark spots at the particle periphery remains unclear. Disintegrated QDs can be excluded as the same spots are observed in samples prepared in the absence of QDs and also from the optical properties, vide infra). The dispersion of the QDs in the polymer particles and, in addition, the well-behaved distribution statistics of the QDs in the particles (vide infra) suggest that indeed particle nucleation occurs in the droplets. Studies of the samples tilted in the TEM suggest that the QDs are not located preferentially at the periphery of the particles but likewise in the polymer particle interior. Average polymer particle sizes of 65 to $75 \mathrm{~nm}$ (number weighted) were determined by TEM for entries 2-2, 2-5, and 2-7. By comparison to the particles prepared by secondary dispersion, the particle sizes and the particle shape are more uniform (Fig. 3).

A comparison of the number densities of quantum dots in the polymerization reaction mixture and the polymer particle number densities in the dispersion prepared with the portion of quantum dot-containing polymer particles (from TEM) shows that the quantum dots are encapsulated into the polymer particles efficiently during the polymeri- zation process (entries 2-2, 2-4 and 2-5). That is, an undesired phase separation $[6,7,25,26]$ does not occur to a significant extent. Concerning the number of quantum dots per polymer particles observed by TEM, $n=1: 47 \%, n=2$ : 4\%, $n \geq 3: 3 \%$ (entry 2-2) and $n=1: 26 \%, n=2: 5 \%, n \geq 3$ : $2 \%$ (entry 2-4) were determined. This agrees within experimental error with a statistical distribution. ${ }^{2}$ Higher quantum dot concentrations (entry 2-5. $n=1: 32 \%, n=2$ : $7 \%, n \geq 3: 9 \%$ ) result in some tendency for aggregation.

For the CdSe quantum dots which are not protected by a $\mathrm{ZnS}$ shell, encapsulation in the polymer results in a blue shift of the photoluminescence maximum by $10 \mathrm{~nm}$ (peak wavelength $610 \mathrm{~nm}$ ) vs. the toluene solution. The photoluminescence intensity is reduced by a factor of 2 to 3 vs. toluene solutions of similar concentration (Fig. 4 and Table 2). For shell-protected $\mathrm{CdSe} / \mathrm{ZnS}$ quantum dots, encapsulation in the polymer has a less pronounced effect on their optical properties, as expected. The emission peak maximum is blue-shifted by 3 to $646 \mathrm{~nm}$. The only slight blue-shift of fluorescence of the polymer-encapsulated QDs is in agreement with their nonaggregated nature: it was reported previously that the energy transfer from higher band gap QDs to smaller band gap QDs resulted in a redshift in aggregates of QDs [25, 27].

\footnotetext{
${ }^{2}$ See footnote 1 .
} 
By comparison to toluene solutions of the same quantum dot particle number density as employed in the polymer particle preparation, emission intensities of the dispersions prepared by polymerization in emulsion are approximately three- to fourfold lower.

In preliminary studies, the intrinsic time-resolved blinking of polymer-encapsulated single quantum dots was observed by confocal microscopy (Fig. 5). Bright photoemission from single polymer-encapsulated quantum dots could be detected (Fig. 5, small bright dots in a and spectrum in b). In timeresolved observations, blinking of the photoluminescence emission is observed (Fig. 5c and d), which is indicative of the individual emitters indeed being single isolated quantum dots [28, 29].

\section{Summary and conclusions}

Polymerization of MMA in the presence of quantum dots miniemulsified in toluene droplets afforded dispersions of PMMA particles with embedded quantum dots. The reaction conditions were such that the solubility of MMA in the aqueous phase exceeded the amount of MMA present. The inorganic nanocrystals are present largely as a single quantum dot embedded in a polymer particle. An undesired aggregation of quantum dots during the polymerization process, which would result in polymer particles with a multiple number of incorporated quantum dots, does not occur to a significant extent. Polymer particles with embedded inorganic nanocrystals were also obtained via a secondary dispersion approach employing premade polymer. The incorporation efficiency of quantum dots was lower in this case, however. Preliminary studies of the photoluminescence of the polymer embedded inorganic nanocrystals confirm that single isolated quantum dots can be addressed.

Acknowledgements Y.G. is grateful for a research stipend by the Alexander von Humboldt-Foundation. We thank Dr. Marina Krumova for TEM analysis. Financial support by the DFG (SFB) is gratefully acknowledged.

\section{References}

1. Bourgeat-Lami E (2004) In: Nalwa HS (ed) Encyclopedia of nanoscience and nanotechnology, vol. 8. American Scientific, Stevenson Ranch, p 305

2. Landfester K, Antonietti M (2002) Prog Polym Sci 27:689

3. Landfester K (2006) Annu Rev Mater Res 36:231

4. Wiese H, Xue Z, Leuninger J (2005) Chem Unserer Zeit 39:65

5. Monteil V, Stumbaum J, Thomann R, Mecking S (2006) Macromolecules 39:2056-2062

6. Fleischhaker F, Zentel R (2005) Chem Mater 17:1346

7. Joumaa N, Lansalot M, Theretz A, Elaissari A (2006) Langmuir 22:1810

8. Alivisatos AP (1996) Science 271:933

9. Qu L, Peng X (2002) J Am Chem Soc 124:2049

10. Talapin DV, Rogach AL, Kornowski A, Haase M, Weller H (2001) Nano Lett 1:207

11. Hines MA, Guyot-Sionnest PJ (1996) J Phys Chem 100:468

12. Dabbousi BO, Rodriguez-Viejo J, Mikulec FV, Heine JR, Mattoussi H, Ober R, Jensen KF, Bawendi MG (1997) J Phys Chem B 101:9463

13. Dubois F, Mahler B, Dubertret B, Doris E, Mioskowski C (2007) J Am Chem Soc 129:482

14. Wisher AC, Bronstein I, Chechik V (2006) Chem Commun 15:1637

15. Selvan ST, Tan TT, Ying JY (2005) Adv Mater 17:1620

16. Darbandi M, Thomann R, Nann T (2005) Chem Mater 17:5720

17. Yang Y, Wen Z, Dong Y, Gao M (2006) Small 2:898

18. Yin W, Liu H, Yates MZ, Du H, Jiang F, Guo L, Krauss TD (2007) Chem Mater 19:2930

19. Darcy D, Dearden A, Masala O, Pickett NL, Ryley S, Sutherland A (2003) Chem Commun 20:2532

20. Kahl M, Thomay T, Kohnle V, Beha K, Merlein J, Hagner M, Halm A, Ziegler J, Nann T, Fedutik Y, Woggon U, Artemyev M, PerezWillard F, Leitenstorfer A, Bratschitsch R (2007) Nano Lett 7:2897

21. Merlein J, Kahl M, Zuschlag A, Sell A, Halm A, Boneberg J, Leiderer P, Leitenstorfer A, Bratschitsch R (2008) Nature Photonics 2:230

22. Landfester K, Montenegro R, Scherf U, Guentner R, Asawapiron SP, Neher D, Kietzke T (2002) Adv Mater 12:651

23. Chai XS, Hou QX, Schork FJ (2006) J Appl Polym Sci 99:1296

24. Chen H, Wagner J (1994) J Chem Eng Data 39:475

25. Lee BH, Kwon KW, Shim M (2007) J Mater Chem 17:1284

26. Csetneki I, Faix MK, Szila A, Gyi S, Kovacs AL, Nemeth Z, Zrinyi M (2004) J Polym Sci A 42:4802

27. Koole R, Liljeroth P, Donega CM, Vanmaekelbergh D, Meijerink A (2006) J Am Chem Soc 128:10436

28. Nirmal M, Dabbousi BO, Bawendi MG, Macklin JJ, Trautman JK, Harris TD, Brus LE (1996) Nature 383:802

29. Gomez DE, Embden JV, Jasieniak J, Smith TA, Mulvaney P (2006) Small 2:204 